\title{
CAN CPAP BE AN OPTION FOR TREATING TINNITUS PATIENTS WITH OBSTRUCTIVE SLEEP APNEA SYNDROME? REVIEW
}

\author{
Alexander Gunenkov, Ekaterina Pchelenok, Sergey Kosyakov \\ National Medical Academy of Continuous Professional Education
}

\begin{abstract}
Tinnitus is the perception of phantom sound in the absence of an external source. Its influence on quality of life varies from minimal to severe. Tinnitus has considerable heterogeneity, thus no single mechanism explains it. There is neither effective drug treatment for the vast majority of patients nor standard rehabilitation management. Keeping that in mind, any reliable option for treatment, even for a minor group, is worth investigating.

The possible relationship between obstructive sleep apnea syndrome and tinnitus, and the effect of continuous positive airway pressure on organ of hearing are discussed.

We have reviewed scientific literature using Medline, PubMed, Google, and conducted a manual search of reference lists of any incorporated paper. The key terms used were: "tinnitus", "obstructive sleep apnea syndrome," and "CPAP".

The following investigation can clarify the tinnitus prevalence in patients suffering from OSAS, specify whether tinnitus is the result of OSAS or its comorbid condition or just a coincidence, and assess the efficacy and indications of this approach to tinnitus treatment.
\end{abstract}

Keywords: tinnitus, CPAP, obstructive sleep apnea syndrome

Address for correspondence:

Alexander Gunenkov

National Medical Academy of Continuous Professional

Education

7 Ivankovskoe Shosse Blvd

125367 Moscow

Russia

e-mail:dralexgun@gmail.com

Received: February 24, 2020

Accepted: March 13, 2020

\section{INTRODUCTION}

Tinnitus is the perception of phantom sound in the absence of an external source. Its influence on quality of life varies from minimal to severe. Tinnitus has considerable heterogeneity, thus no single mechanism explains it $(1,2)$. There is neither effective drug treatment for the vast majority of patients nor standard rehabilitation management. Keeping that in mind, any reliable option for treatment, even for a minor group, is worth investigating (3). 
In the recent years we have conducted research dealing with tinnitus in patients with obstructive sleep apnea syndrome (OSAS), who use constant positive airway pressure (CPAP) equipment. We expected to correlate our preliminary encouraging results with other studies. An additional reason for our research were the advertisements in CPAP centers for people suffering from tinnitus.

\section{CURRENT RESEARCH GOALS AND RESULTS}

The literature search included Medline, PubMed, Google, and a manual search of reference lists of any incorporated paper. It was conducted using "tinnitus", "obstructive sleep apnea syndrome", and "CPAP" as three key terms. The final search was conducted in March 2020.

We found a few papers $(4,5,6)$ concerning tinnitus in people suffering from obstructive sleep apnea syndrome.

The paper of Taiwanese colleagues concerning tinnitus in patients with OSAS (4) included 21,798 middle-aged and elderly patients with otolaryngologist-diagnosed tinnitus from the Longitudinal Health Insurance Database 2000 of the Taiwan National Health Insurance Research Database. A total of 108,990 controls were also identified from the same database based on frequency-matching on 10year age interval, sex, and year of index date of the cases. In this population-based, case-control study, the risk of tinnitus was found to be significantly higher among patients with sleep apnea compared to those without the condition.

Keeping in mind the lack of papers we manually added the piece of information concerning directly the link between CPAP and tinnitus treatment: Dr. J. T. Lai's report during the $1^{\text {st }}$ World Tinnitus Congress, 22-24 May 2017. His data also indicated high prevalence of OSAS among tinnitus patients who have sought help in his tinnitus centre (5). Short-term outcome for CPAP treatment concerning tinnitus seems to be positive (6).

The probable links between tinnitus and OSAS are obscure so far. Perhaps they are the following:

1. OSAS can cause hearing loss, which consequently provokes tinnitus.
Nonetheless, there is discrepancy in the papers dealing OSAS influence on hearing.

J.H. Huwang et al. (7) studied 34 OSA subjects and 190 control subjects and reported no significant positive association with averaged pure-tone thresholds (PTA), neither low frequency nor high PTA.

E. Matsumura et al. (8) obtained the same results concerning PTA in 38 adult males divided into groups matched for age and Body Mass Index. There was no difference between the groups in terms of hearing thresholds, tympanometry and evaluated brainstem auditory evoked response (BAER) parameters. An association was observed between the presence of obstructive sleep apnea and changes in absolute latency of wave V. There was an association between moderate obstructive sleep apnea and change of the latency of wave V. The authors' conclusions were:

1. The presence of obstructive sleep apnea is associated with changes in nerve conduction of acoustic stimuli in the auditory pathway in the brainstem;

2. The increase in obstructive sleep apnea severity does not promote worsening of responses assessed by audiometry, tympanometry and BAER.

On the contrary, C. Casale et al. (9) found that patients (39 OSA persons) with OSAS had a PTA significantly higher than the control group (21 subjects) with snoring without OSAS. Analyzing the single frequencies, the OSAS group showed higher thresholds and was statistically significant at $4 \mathrm{kHz}$ compared to the control group. In addition to PTA differences, lower transient evoked otoacoustic emissions (TEOAE) reproducibility and distortion product otoacoustic emissions (DPOAE) amplitude, the mean latencies of waves I, III, and V were prolonged in the OSAS group as compared to the healthy people. These findings suppose that severe OSAS could represent a risk factor for an auditory pathway.

M. S. Gozeler et al. (10) conducted a study involving 35 patients diagnosed with moderate or severe OSAS, and a control group consisting of 30 individuals. The authors determined a mild sensorineural hearing loss in patients with OSAS. When the different frequencies were evaluated separately, hearing threshold values in the patients with OSAS were 
significantly higher compared to the control group at $500,1000,2000,4000$, and $8000 \mathrm{~Hz}$ in both ears. TEOAE test reproducibility values in both ears were significantly lower in the study group compared to the control group.

S. Ekin et al. (11) did not reveal hearing loss at low and high frequencies, but identified it at extended high frequencies $(>8 \mathrm{kHz}$ ). The authors found a significant correlation between the duration below $90 \%$ saturation and mean saturation and high-frequency hearing loss, but did not find a correlation between the apnea-hypopnea index and high-frequency hearing loss. The authors established a significant difference between the 2 groups (control vs. simple snoring and OSA patients); however, no difference between OSA patients and the simple snoring group in high-frequency hearing loss was found. So the authors believe that hearing loss may be due to continuous noise exposure rather than hypoxia in patients with OSA.

A. Chopra et al. (12) observed a dose-response relationship between nadir $\mathrm{SpO} 2$ and the severity of hearing impairment where decreasing nadir $\mathrm{SpO} 2$ was associated with increasing hearing loss severity, supporting hypoxic injury as a linking mechanism between sleep apnea and hearing loss. They also found that more severe OSAS was associated with more severe hearing impairment. This suggests a dose response relationship between OSAS and hearing loss.

F. Martines et al. (13) examined one hundredsixty patients that underwent overnight polysomnography, micro-otoscopy, multi-frequency audiometry, acufenometry, and TEOAE. All subjects were divided into four groups based on presence/absence of following parameters: simple snoring without OSAS, mild OSAS, moderate OSAS, severe OSAS. All groups were characterized by a mean hearing threshold $<25 \mathrm{~dB}$ HL for $0.25-3 \mathrm{kHz}$ frequencies and a progressive decrease in hearing sensitivity, particularly for $6-16 \mathrm{kHz}$ frequencies. The analysis of otoacoustic emissions evidenced a significant difference between simple snoring and severe OSAS individuals for 3 and $4 \mathrm{kHz}$ frequencies. The results underline the key role of chronic nocturnal intermittent hypoxia in the development of an early cochlear dam- age and a more marked high-frequency hearing loss in case of severe OSAS.

Deniz et al. (14) figured out in an audiological assessment of 160 participants divided into groups according to the severity of the apnea-hypopnea index and blood oxygen saturation that all patients in the control group and in mild OSAS group had normal hearing thresholds (lower than 26dB). The patients who had moderate and severe OSAS had varying degrees of sensorineural hearing losses.

Summing up this information, the conclusions of these papers vary from no impact of OSAS to hearing loss, its influence on exclusively high or PTA $>8$ $\mathrm{kHz}$ frequency, to more prominent damage.

2. OSAS-induced hypoxia can directly effect on organ of hearing and cause tinnitus onset and persistence (15). The authors conclude that the lowest oxyhemoglobin saturation may be an independent factor influencing auditory function in severe obstructive sleep apnea. The results of the aforementioned paper (8) support this point of view.

3. OSAS can impair sleep quality, which could cause tinnitus. It is well established that tinnitus severity is related to sleep quality $(4,16)$. We discovered a paper concerning sleep quality, particularly before tinnitus onset (17), suggesting that the sleep disturbances may have an impact on tinnitus occurrence and persistence.

M. Koo and J. H. Hwang, in the aforementioned paper (4), concluded that the risk of tinnitus was higher in patients with sleep disturbance compared to those without the condition, and the risk of tinnitus was higher in patients with sleep apnea compared to those without the condition. In this population-based, case-control study, the risk of tinnitus was found to be significantly higher among middleaged and elderly patients with sleep disturbances, especially with sleep apnea.

Contemporary view for tinnitus development and persistence focuses on the reorganization of hearing and non-hearing structures of the central nervous system $(18,19,20)$, with emergence of new abnormal links. As far as OSAS and tinnitus are concerned, the mechanism could consist of a) worsening of hearing that could be identified by PTA shift and OAE recordings, b) sleep problems, c) direct impact to brain including central structures of hearing organ 
(like the genesis of somatosensory tinnitus caused by temporomandibular disorders). All aforementioned ways are possible as well as their combinations.

With these considerations, CPAP positive impact on tinnitus can emerge in different means:

3. The compensation of OSAS-induced hypoxia;

4. Improving sleep quality;

5. Last but not least: we do consider that noise induced by CPAP equipment could be helpful in avoiding silence and hence beneficial to tinnitus masking.

However, CPAP has negative side effects on the organ of hearing that should be considered. Among them are:

a) A significant increase of the middle ear pressure in patients using CPAP regularly for 6 months. This increase is proportional to the pressure level of the CPAP device (21).

b) There is the paper concerning the clinical case of a patient with otic barotrauma as a result of excessive self-titration of CPAP in an in-home setting (22).

c) A rare case of vertigo secondary to CPAP. To date, there has been only one report of hearing loss and vertigo during such treatment, fortunately with complete recovery (23).

d) In the aforementioned report J. T. Lai (6) spoke about 3 patients among 14 whose tinnitus had worsened.

\section{CONCLUSION}

The following investigation can clarify the tinnitus prevalence in patients suffering from OSAS, specify whether tinnitus is the result of OSAS or its comorbid condition or just a coincidence, and assess the efficacy and indications of this approach to tinnitus treatment.

\section{REFERENCES}

1. Elgoyhen $A B$, Langguth $B$, De Ridder D, Vanneste

$S$. Tinnitus: perspectives from human neuroimaging. Nat Rev Neurosci. 2015; 16(10):632-42. doi: $10.1038 /$ nrn 4003 .

2. Langguth B, Landgrebe M, Schlee W, Schecklmann M, Vielsmeier V, Steffens T, et al. Different patterns of hearing loss among tinnitus patients: A latent class analysis of a large sample. Front Neurol. 2017;8:46. doi: 10.3389/fneur.2017.00046.
3. Hall D, Láinez M, Newman C, Sanchez T, Egler M, Tennigkeit $\mathrm{F}$, et al. Treatment options for subjective tinnitus: Self reports from a sample of general practitioners and ENT physicians within Europe and the USA. BMC Health Serv Res. 2011; 11:302. doi: 10.1186/1472-6963-11-302.

4. Koo M, Hwang JH. Risk of tinnitus in patients with sleep apnea: A nationwide, population-based case control study: Tinnitus in patients with sleep apnea. Laryngoscope. 2016; 127(9):2171-5. doi: 10.1002/lary.26323.

5. Lai JT, Lin CY, Yan YH, Shen PH. High prevalence of obstructive sleep apnea syndrome in patients with tinnitus. J Hear Sci. 2017; 7(2):109.

6. Lai JT. Continuous positive airway pressure (CPAP) treatment outcome in chronic tinnitus of unknown: A preliminary study. J Hear Sci. 2017; 7(2):108.

7. Hwang JH, Chen JC, Hsu CJ, Liu TC. Association of obstructive sleep apnea and auditory dysfunctions in older subjects. Otolaryngol Head Neck Surg. 2011;144(1):114-19. doi: 10.1177/0194599810390859.

8. Matsumura E, Matas CJ, Magliaro FC, Pedreño R. Evaluation of peripheral auditory pathways and brainstem in obstructive sleep apnea. Braz J Otorhinolaryngol. 2016. pii: S1808-8694(16)30233-6. doi: 10.1016/j.bjorl.2016.10.014.

9. Casale M, Vesperini E, Potena M, Pappacena M, Bressi F, Baptista PJ, et al. Is obstructive sleep apnea syndrome a risk factor for auditory pathway? Sleep Breath. 2012; 16(2):413-7. doi.org/10.1007/ s11325-011-0517-x.

10. Gozeler MS, Kilic K, Sakat MS, Sengoz F. Auditory functions of patients with obstructive sleep apnea syndrome. Eurasian J Med. 2019. doi: 10.5152/ eurasianjmed.2019.18373.

11. Ekin S, Turan M, Arısoy A, Gunbatar H, Sunnetcioglu A, Asker S, et al. Is there a relationship between obstructive sleep apnea (OSA) and hearing loss? Med Sci Monit. 2016;22:3124-8. doi:10.12659/ msm.897347.

12. Chopra A, Jung M, Kaplan RC, Appel DW, Dinces EA, Dhar S, et al. Sleep apnea is associated with hearing impairment: The Hispanic Community Health Study/Study of Latinos. J Clin Sleep Med. 2016;12(5):719-26. doi:10.5664/jcsm.5804

13. Martines F, Ballacchino A, Sireci F, Mucia M, Mattina E, Rizzo S, et al. Audiologic profile of OSAS 
and simple snoring patients: the effect of chronic nocturnal intermittent hypoxia on auditory function. Eur Arch Otorhinolaryngol. 2016; 273(6):1419-24. doi: 10.1007/s00405-015-3714-6.

14. Deniz M, Çiftçi Z, Ersözlü T, Gültekin E, Alp R. The evaluation of auditory system in obstructive sleep apnea syndrome (OSAS) patients. Am J Otolaryngol. 2016;37(4):299-303. doi: 10.1016/j. amjoto.2016.03.004.

15. Seo YJ, Park SY, Chung HJ, Kim CH, Lee JG, Kim $\mathrm{SH}$, et al. Lowest Oxyhemoglobin Saturation May Be an Independent Factor Influencing Auditory Function in Severe Obstructive Sleep Apnea. J Clin Sleep Med. 2016;12(5):653-8. doi: 10.5664/ jcsm. 5786.

16. Crönlein T, Langguth B, Geisler P, Hajak G. Tinnitus and insomnia. Prog Brain Res. 2007;166:227-33. doi: 10.1016/S0079-6123(07)66021-X.

17. Lu T, Li S, Ma Y, Cheng Z. Positive correlation between tinnitus severity and poor sleep quality prior to tinnitus onset: a retrospective study. Psychiatr Q. 2020. doi: 10.1007/s11126-019-09708-2.

18. Eggermont JJ, Roberts LE. The neuroscience of tinnitus. Trends Neurosci. 2004; 27(11):676-82. doi: 10.1016/j.tins.2004.08.010
19. De Ridder D, Elgoyhen AB, Romo R, Langguth B. Phantom percepts: tinnitus and pain as persisting aversive memory networks. Proc Natl Acad Sci USA. 2011; 108(20):8075-80. doi: 10.1073/ pnas.1018466108.

20. Landgrebe $M$, Langguth $B$, Rosengarth $K$, Braun S, Koch A, Kleinjung T, et al. Structural brain changes in tinnitus: grey matter decrease in auditory and non-auditory brain areas. Neuroimage. 2009;46(1):213-8. doi: 10.1016/j. neuroimage.2009.01.069.

21. Sivri B, Sezen OS, Akbulut S, Coskuner T. The effect of continuous positive airway pressure on middle ear pressure. Laryngoscope. 2013;123(5):1300-4. doi: 10.1002/lary.23896.

22. McCormick J, Hildrew D, Lawlor C, Guittard J, Worley N. Otic barotrauma resulting from continuous positive airway pressure: Case report and literature review. Ochsner J. 2016; 16(2): 146-9. doi: 10.5664/jcsm.6630.

23. Endara-Bravo A, Ahoubim D, Mezerhane E, Abreu RA. Alternobaric vertigo in a patient on positive airway pressure therapy. J Clin Sleep Med. 2013;9(12):1347-8. doi: 10.5664/jcsm.3288. 